\title{
Auditoría de estados financieros en CONTEXTO DE PANDEMIA POR COVID-19: UN ANÁlISIS DE LA NORMATIVA
}

ARGENTINA

\author{
Audit of FINANCIAL STATEMENT IN THE CONTEXT OF THE \\ COVID-19 PANDEMIC: AN ANALYSIS OF THE ARGENTINE RULES
}

Diana Ester Albanese ${ }^{1}$, Claudia Inés Rivera ${ }^{2}$

Fecha recepción: $27 / 10 / 2020$

Fecha aceptación: 22/03/2021

\section{Resumen}

La auditoría basada en riesgos es una forma de conducir las auditorías que incluye una noción ampliada de eventos negativos vinculados con el negocio, los procesos y posibles situaciones de fraude. La situación de pandemia derivada del brote del virus COVID-19 que atraviesa el mundo en general y la Argentina en particular profundiza ciertos riesgos que impactan en las organizaciones y la auditoría de estados financieros. El objetivo del presente trabajo es analizar los riesgos asociados al contexto de pandemia y su efecto en la opinión del auditor en el marco de la normativa vigente en la Argentina. Para ello se realizó un estudio descriptivo basado en la revisión bibliográfica. Las situaciones más relevantes que se presentan al contador son la consideración de aspectos vinculados a la hipótesis de empresa en marcha y las posibles limitaciones en el alcance del trabajo del auditor para obtener elementos de juicio válidos y suficientes que respalden su informe.

Palabras clave: COVID-19, auditoría basada en riesgos, informe del auditor.

1 Departamento de Ciencias de la Administración, Universidad Nacional del Sur (DCA, UNS). E-mail: dalbanese@uns.edu.ar. ORCID: https://orcid.org/0000-0002-9462-5356.

2 Departamento de Ciencias de la Administración, Universidad Nacional del Sur (DCA, UNS). E-mail: claudiarivera@bvconline.com.ar. ORCID: https:/ / orcid.org/0000-0002-5746-6157. 


\begin{abstract}
Auditing based on risks includes a broader definition of the business risks and processes, and a greater attention to fraud risk. The pandemic situation derived from the COVID-19 outbreak taking place in the world in general and in Argentina in particular, looks into certain risks and introduces new events that impact on the financial statement audit. From a risk-based auditing approach, the present article aims to identify the risks associated to the pandemic context and the impact on the auditor's opinion. A descriptive study was carried out based on the bibliographic review. The most relevant situations submitted to the accountant are the assessment of the on-going enterprise scenario and the possible limitations to obtain valid and sufficient evidence during his job development.
\end{abstract}

Keywords: COVID 19, auditing based on risks, audit report.

JEL: M41. 
Auditoría de estados financieros en contexto de pandemia por COVID-19: ...

\section{Introducción}

En el desarrollo del encargo de auditoría, el contador debe considerar el riesgo de auditoría entendido como la posibilidad de que los estados financieros contengan desvíos significativos por errores o irregularidades, que excedan el nivel de significación, y que hagan que el auditor emita una opinión equivocada, la cual es relevante para los usuarios de la información al momento de tomar decisiones.

La Norma ISO 31000/2009 define al riesgo como la posibilidad de que ocurra un evento que tenga impacto negativo sobre el logro de los objetivos; es decir, es el efecto de la incertidumbre en la consecución de los mismos. Casal (2010) manifiesta que el riesgo es la exposición a las consecuencias de la incertidumbre sobre variaciones respecto de lo planeado o esperado, pudiendo resultar tanto una pérdida como un beneficio. El autor sostiene que, considerando la incertidumbre como un elemento subyacente, es posible reducir el riesgo a través del establecimiento de sistemas y diseño de acciones que minimicen sus consecuencias negativas y maximicen las positivas. En términos generales, el riesgo implica una incertidumbre respecto a eventos y resultados que podrían impactar de manera adversa en los objetivos y metas organizacionales (Muñoz Giró, 2011).

Hace algunos años surgió una forma de conducir la auditoría denominada Auditoría Basada en Riesgos (ABR), la cual incluye una noción ampliada de eventos negativos relevantes que incluye los vinculados al negocio y a los procesos, así como mayor atención a posibles fraudes (Bell, Peecher, Solomon, Marrs \& Thomas, 2007). Se trata de un paso más hacia la calidad de las auditorías. Para ello no solo se debe considerar la normativa vigente y los mejores estándares, sino resaltar la importancia del escepticismo profesional del auditor.

En diciembre de 2019 se conoció la aparición en China de un nuevo coronavirus SARS-CoV-2 (conocido como "COVID-19"), que con posterioridad al 31 de diciembre de 2019 se expandió prácticamente en todo el mundo y el 11 de marzo de 2020 la Organización Mundial de la Salud (OMS) declaró como una pandemia. Esta situación impactó de manera muy significativa en la economía de las organizaciones presentando nuevos riesgos de negocios que deberán ser considerados por los auditores, especialmente en cuanto a la factibilidad de continuar como empresa en marcha y las posibles limitaciones para obtener elementos de juicio válidos y suficientes durante el desarrollo de su trabajo.

Sin lugar a dudas, la situación de pandemia que atraviesa el mundo en general y la Argentina en particular profundiza los riesgos de los encargos de 
auditoría y revisión de estados financieros y presenta nuevas incertidumbres a nivel de las organizaciones y del desarrollo del trabajo que los contadores deberán evaluar. Frente a esta situación de emergencia, en la Argentina, la Federación Argentina de Consejos Profesionales en Ciencias Económicas (FACPCE) publicó en abril del año 2020 una "Guía sobre la aplicación de Normas de Contabilidad y Auditoría en el contexto COVID-19", en la cual se brindan pautas para el tratamiento de aspectos contables y de auditoría afectados por el contexto.

De acuerdo a lo expuesto anteriormente, el presente trabajo tiene por objetivo analizar la normativa vigente en la Argentina sobre la consideración de los riesgos de auditoría en el contexto de pandemia por COVID-19. Para ello se realizó un estudio descriptivo basado en la revisión bibliográfica, en el cual se examina la "Guía sobre la aplicación de Normas de Contabilidad y Auditoría en el contexto COVID-19" (FACPCE, 2020) en forma conjunta con las normas de contabilidad y auditoría vigentes en la República Argentina, ya que la mencionada guía no conforma nueva normativa, sino una orientación para la aplicación de la existente. Además de ello se consideraron las disposiciones del international standard on auditing (ISA) emitidas por el international auditing and assurance standards board (IAASB)

Para abordar el objetivo propuesto, el trabajo se estructura de la siguiente manera. En primer lugar, en la sección 2 se aborda el concepto de auditoría basada en riesgos. Luego, en el apartado 3 se analizan aquellos eventos derivados de la situación de pandemia que influyen en el desarrollo del encargo de auditoría y en la sección 3 se examina su impacto en el informe del auditor. Finalmente, en las consideraciones finales se discuten los temas abordados y se presentan futuras líneas de investigación.

\section{Auditoría basada en riesgos}

Las mejores prácticas de auditoría siempre tuvieron en cuenta la evaluación del riesgo en la planificación del encargo. No obstante, su concepción fue evolucionando. Pasando de considerar el riesgo combinado inherente y de control y el riesgo de detección - componentes del riesgo de auditoría - a incorporar en el modelo de auditoría basada en riesgos un análisis estratégico, considerando el riesgo del negocio (businessrisk), valorando la probabilidad de ocurrencia y el posible impacto en los estados financieros de las declaraciones equivocadas materiales (Casal, 2013) y los riesgos de los procesos y dando mayor atención 
a posibles situaciones que deriven en fraudes.

Tal como se dijo, la ABR considera no solo los estados financieros, sino los procesos, sistemas de información y el sistema de gestión, y su planificación y desarrollo pone énfasis en los riesgos críticos que puedan causar mayor impacto negativo en la consecución de los objetivos de la organización: estratégicos, operacionales, de información y cumplimiento, con el propósito de confirmar si las operaciones se corresponden con la estrategia del negocio, las buenas prácticas de control interno y normas legales aplicables (Lattuca, 2014). En este enfoque estratégico, el auditor no solo evalúa hechos del pasado comparándolos con ciertos parámetros, ni se limita a hacer recomendaciones sobre el sistema de control interno, sino que agrega valor a la organización identificando eventos negativos que afectan su actividad.

Las organizaciones van cambiando hacia un escenario en el cual la administración de riesgos, mediante variadas metodologías, se torna necesaria para su subsistencia. Por las razones expresadas en los apartados anteriores, se considera que la ABR agrega valor evaluando específicamente los riesgos detallados a continuación.

\section{a. Riesgo del negocio}

Se entiende por riesgo del negocio la probabilidad de que un evento o acción afecte en forma adversa la capacidad de la organización para lograr los objetivos empresariales y/o llevar a cabo sus estrategias en forma positiva (Casal, 2013). El auditor debe evaluar los factores de riesgo que afectan cada negocio, y los efectos que potencialmente pueden acarrear sobre los estados financieros. Pueden tener distintas fuentes: ambiente externo de la organización (el entorno: proveedores, clientes, mercados, estado, etc., y agruparse en microentorno y macroentorno); ambiente de la industria de la organización (el entorno competitivo, los factores que influyen en un tipo de negocios determinado; el grupo de empresas que producen bienes o servicios sustitutos entre sí); ambiente interno, entre otros (Albanese \& López, 2017). En el enfoque basado en riesgos el auditor, tal como se dijo, debe entender la ventaja competitiva del cliente: sus nichos, su situación frente a los competidores, los planes para crear valor, y conocer las amenazas al cumplimiento de los objetivos del negocio. Asimismo, debe conocer si el cliente tiene implementado un proceso de administración de riesgos y su efectividad. El contador debe identificar los eventos que puedan afectar el negocio y que deriven en errores significativos 
en los estados financieros para determinar el grado adecuado de la evidencia de auditoría (Arens, Elder \& Beasley, 2007).

Tanto la literatura como la normativa internacional, en particular la ISA n. ${ }^{\circ} 315$ revisada (IAASB, 2019), guían a los auditores a considerar el riesgo de negocio del cliente cuando evalúan el riesgo de error material durante la fase de planificación de la auditoría financiera (Mantilla Blanco \& Casal, 2012; Schultz, Bierstaker \& Donell, 2010); por el contrario, según Casal (2013) no se puede afirmar que las normas argentinas necesariamente provean lineamientos a ese fin.

\section{b. Riesgos de los procesos}

Una vez evaluados los riesgos del negocio, el auditor debe entender los procesos clave; identificar los eventos que los amenazan y la efectividad de los controles de los procesos para hacerles frente. En todos los casos, el entendimiento de los procesos es parte fundamental de la comprensión del riesgo del negocio (Arens, Elder \& Beasley, 2007).

Los riesgos de procesos se identifican como todas aquellas situaciones que afectan o impiden el cumplimiento del objetivo del proceso. Es decir, consiste en identificar aquellas situaciones por las cuales el proceso no funciona de acuerdo al objetivo definido por la organización y los factores que puedan hacer que el riesgo se materialice.

\section{c. Riesgo del encargo de auditoría}

En general, la bibliografía aborda la administración de riesgos sobre los procesos organizacionales pero no se profundiza en el desarrollo de la auditoría, que es relevante para garantizar la calidad de la misma a pesar de estar íntimamente vinculados.

La falta de identificación de riesgos en cada subproceso de la auditoría llevará a un deterioro de su calidad, y como consecuencia a que el auditor emita una opinión equivocada sobre la información financiera (Hernández Melendrez \& Sánchez Gómez, 2016). En consecuencia, el contador deberá estudiar en cada organización la estrategia de la cadena de valor al momento de realizar su planificación. Por lo indicado, queda expuesta la vinculación entre las medidas que tomen las organizaciones para identificar y gestionar sus riesgos y el proceso de auditoría. 


\section{Impacto de la pandemia en los encargos de auditoría}

La declaración de la pandemia por parte de la OMS y las medidas que se han tomado para hacerle frente provocaron una paralización temporal de la actividad económica y un efecto negativo en la economía, cuya duración y cuantificación es incierta. La FACPCE (2020) publicó una guía sobre aplicación de normas de contabilidad y auditoría que brinda pautas para el tratamiento de aspectos contables y de auditoría afectados por el contexto y donde se mencionan situaciones de riesgos adicionales en la preparación de estados financieros, tales como: posibilidad de que el valor en libros de activos y pasivos requiera ajustes en un período posterior; necesidad de reconocer nuevos pasivos; o revelación en notas de nuevas situaciones contingentes. Los nuevos eventos derivados de una situación externa extraordinaria y desconocida deben ser afrontados por las organizaciones en general y los auditores en particular.

\subsection{Efectos sobre el análisis de riesgos}

En la consideración de los riesgos del negocio, el auditor deberá evaluar de qué manera la pandemia y las medidas que se han tomado para hacerle frente han afectado el negocio de la entidad y su ambiente. Cada empresa, por intermedio de su dirección o gerencia, debería hacer su propia evaluación en cuestiones tales como suministro de proveedores con zonas afectadas, continuidad de la operatoria de la entidad en caso de que el personal se infecte, fluctuaciones de los precios de mercado y los impactos que eso tendría sobre los flujos de efectivo. El auditor se basará en el análisis hecho por la dirección de la entidad y en su propio juicio, mediante procedimientos de indagaciones a la dirección, lectura de publicaciones específicas, análisis de información contable posterior al cierre y proyecciones reformuladas de la dirección, entre otros procedimientos que le permitan comprender los efectos negativos en el negocio del cliente (Arens, Elder \& Beasley, 2007).

El riesgo de fraude es incorporado como un componente del riesgo del proceso de auditoría, donde los profesionales deben centrarse en las posibles irregularidades tanto en la auditoría de control interno como en la de estados financieros. La consecuencia para la auditoría basada en riesgos es lograr una perspectiva de riesgo centrada en el riesgo de fraude (Casal, 2010). La ISA n. 240 (IAASB, 2013) considera que, a pesar de que el fraude es un concepto jurídico amplio, al auditor le compete aquel que da origen a distorsiones significativas en los 
estados financieros, las cuales pueden derivar de la presentación fraudulenta de información financiera, y/o la apropiación indebida de bienes.

En la situación motivada por la pandemia, el auditor deberá estar atento a cómo el nuevo ambiente de trabajo y las acciones tomadas por la entidad podrían generar nuevas situaciones de fraudes. A modo de ejemplo, se pueden citar algunas situaciones:

- Frente a una disminución significativa de los ingresos, la entidad podría verse tentada a manipular cierta información contable para alcanzar presupuestos o ratios comprometidos.

- La modalidad de trabajo a distancia podría obviar ciertos controles establecidos por la entidad en beneficio de algún funcionario o empleado.

- Podrían activarse gastos vinculados a la pandemia que deberían imputarse como pérdida u omitir la contabilización de provisiones (por ejemplo, por contratos onerosos) para mejorar los resultados.

- No informar adecuadamente en notas a los estados financieros situaciones relacionadas con dudas sobre la capacidad de la entidad para continuar como empresa en marcha.

- Posibles compras sin haberse seguido el debido proceso previo que concluya con la selección de la oferta más conveniente para la entidad, favoreciendo a algún empleado del sector para que obtenga un beneficio indebido.

El riesgo del encargo de auditoría, previamente evaluado por el auditor, puede haberse incrementado, aun en una auditoría recurrente. El auditor deberá considerar posibles alteraciones de la información contable por parte de la dirección producto de la alteración de la gestión financiera causada por la pandemia. Otro riesgo que puede afectar el trabajo del auditor es el cambio en la modalidad operativa: el trabajo del personal administrativo en forma remota desde sus hogares podría haber disminuido la ejecución de ciertos controles, lo cual obliga al contador a revisar la planificación de su tarea (FACPCE, 2020).

El auditor tendrá que reconsiderar la estrategia, diseñando y ejecutando procedimientos de auditoría adicionales para responder a esos nuevos riesgos que podrían derivar en incorrecciones significativas, ya sea por error o fraude. 


\subsubsection{Reconsideración de la validez de la hipótesis de empresa en marcha}

Este es uno de los aspectos más relevantes que el auditor debe considerar como consecuencia de la situación de pandemia. La normativa nacional aclara que será de aplicación a la preparación de estados financieros el principio de empresa en marcha definido como empresa que está en funcionamiento y continuará sus actividades dentro del futuro previsible, excepto que existan razones por las cuales se ponga en duda dicha situación. En este caso, se deben exponer específicamente los criterios utilizados para la elaboración de los estados financieros y las razones por las cuales se llegan a estas conclusiones (RT n. ${ }^{\circ} 17$ [FACPCE, 2000]; RT n. ${ }^{\circ} 41$ [FACPCE, 2015]).

A nivel internacional, la Norma Internacional de Contabilidad (NIC) 1 (International Accounting Standards Board [IASB], 2008) establece, en relación con el principio de empresa en marcha, que la entidad debe elaborar sus estados financieros bajo la hipótesis de continuidad excepto que se pretenda liquidar la empresa o cesar su actividad. Asimismo, establece que:

Cuando la gerencia, al realizar esta evaluación, sea consciente de la existencia de incertidumbres importantes, relativas a eventos o condiciones que puedan aportar dudas significativas sobre la posibilidad de que la entidad siga funcionando normalmente, procederá a revelarlas en los estados financieros. Cuando una entidad no prepare los estados financieros bajo la hipótesis de negocio en marcha, revelará este hecho, junto con las hipótesis sobre las que han sido elaborados, así como las razones por las que la entidad no se considera como un negocio en marcha.

La ISA n. ${ }^{\circ} 570$ (IAASB, 2015) establece que el auditor debe obtener evidencia de auditoría válida y suficiente sobre la idoneidad de la utilización por parte de la dirección de la hipótesis de empresa en funcionamiento para la preparación y presentación de los estados financieros, y concluir si existe incertidumbre significativa respecto a la capacidad de la entidad para continuar funcionando. Si el auditor concluye que la aplicación del principio de empresa en marcha es adecuada, deberá analizar si pese a la existencia de una incertidumbre significativa existe una adecuada revelación en los estados financieros de los principales hechos que puedan generar dudas sobre la continuidad de funcionamiento del ente y los planes de la dirección para afrontarlos (Albanese \& Bonifazi, 2019). 
Este análisis cobra especial interés en este entorno donde las empresas se ven especialmente afectadas por el impacto negativo que la pandemia tiene sobre la economía en general, generando muchas incertidumbres. En las actuales circunstancias, es esencial que el auditor aumente su escepticismo profesional y procure obtener elementos de juicio válidos y suficientes sobre los cuales basar su opinión (FACPCE, 2020).

Mediante la indagación a funcionarios y empleados del ente, el auditor tratará de identificar si existen dudas sustanciales sobre la capacidad del ente para continuar como una empresa en funcionamiento durante un período que debe ser al menos de doce meses posteriores a la fecha de cierre de los estados contables. El auditor indagará acerca de:

- Los aspectos importantes que puedan afectar la información contenida en los estados, identificando aquellas afirmaciones que podrían tener un riesgo de incorrección incrementado como consecuencia de la pandemia y las medidas tomadas.

- Los cambios que la situación y las medidas tomadas han producido en las actividades de negocios de la entidad, su ambiente y la industria.

- Cuáles han sido los impactos directos e indirectos de la situación económica, qué segmentos o actividades se han visto afectadas negativa o positivamente.

- Cómo están operando sus proveedores críticos.

- El cumplimiento de sus obligaciones.

- Cómo se ha visto afectada la rotación de sus cuentas por cobrar y los riesgos de recupero.

- Si ha cambiado su evaluación de la capacidad de la entidad para continuar como una empresa en marcha durante un período al menos de doce meses posteriores a la fecha de cierre de los estados financieros del período bajo revisión.

Este conocimiento le posibilitará al auditor una mejor interpretación de las variaciones observadas en la información contable entre el período bajo revisión y períodos anteriores comparables, al realizar su revisión analítica. La información recogida también será útil para identificar cualquier modificación significativa de medición y/o de exposición en los estados financieros, específicamente relacionadas con los efectos de la pandemia. 


\subsubsection{Limitaciones en el alcance del trabajo del auditor}

La aplicación de ciertos procedimientos destinados a obtener evidencias de auditoría puede verse alterados por la situación de pandemia. Podrían surgir distintos problemas que el auditor deberá intentar resolver para formarse una opinión o conclusión sobre los estados financieros en su conjunto y emitir su informe de auditoría o de revisión, sin afectar la calidad de su trabajo profesional. A continuación, se enumeran algunas situaciones que pueden impedir el desarrollo de algunos procedimientos de auditoría:

\section{a. Evaluación del control interno pertinente a la valoración del riesgo}

Si bien la Resolución Técnica (RT) n. ${ }^{\circ} 37$ (FACPCE, 2013) ${ }^{3}$ en la enumeración de los procesos de auditoría incluye la evaluación del sistema de control interno, el relevamiento de su diseño y funcionamiento se realiza en la etapa de planificación del encargo a efectos de definir el enfoque de auditoría. La situación de pandemia que atraviesan las organizaciones puede provocar la modificación de los procesos y la desaparición de controles existentes o su debilitación. A modo de ejemplo, se puede mencionar el incremento de riesgos de ataque cibernético, por el teletrabajo amenazando la integridad de la empresa. Por otro lado, la segregación de funciones también podría haberse visto afectada. Ante este nuevo escenario de operaciones, el contador deberá hacer un relevamiento de qué controles clave siguen funcionando tal cual fueron diseñados e identificar aquellos que se han visto afectados analizando su impacto y probabilidad de ocurrencia a efectos de replantear ciertos procedimientos.

3 Se encuentra en período de consulta el Proyecto n. ${ }^{\circ} 43$ de Resolución Técnica "Normas de Auditoría, Revisión, otros Encargos de Aseguramiento, Certificaciones, Servicios Relacionados e Informes de Cumplimiento" aprobado por la Junta de Gobierno de FACPCE el 1 de octubre de 2020 por un período de 180 días. Dicho proyecto fue elaborada por el Consejo Elaborador de Normas de Contabilidad y Auditoría (CENCYA) como propuesta de modificación de la actual Resolución Técnica n. ${ }^{\circ} 37$. 


\section{b. Cotejo de los estados financieros con los registros contables}

En la ejecución de este procedimiento, el contador podría encontrar limitaciones para acceder a los registros contables de la entidad, debido a que el proceso de cierre contable del período que está auditando/revisando pudo haberse modificado por las circunstancias mencionadas. Por su parte, la entidad podría tener dificultades para transcribir los estados financieros al Libro Inventarios y Balances y sus asientos al Libro Diario General. Ante esta situación, el auditor podrá: solicitar a la entidad la inclusión de una nota en sus estados contables; incluir un párrafo adicional en el informe del auditor en la sección de información especial requerida por leyes o disposiciones reglamentarias nacionales, provinciales, municipales o de los organismos públicos de control o de la profesión; incluir, cuando corresponda, párrafos específicos en la carta de representaciones de la gerencia (FACPCE, 2020).

\section{c. Revisión de la correlación entre registros contables y entre estos y la do- cumentación respaldatoria}

El auditor podría encontrarse con restricciones al momento de inspeccionar la documentación de respaldo. Frente a esta situación, podrá solicitar al cliente documentación por vía digital (e-mails, imágenes, fotos, filmaciones, documentación escaneada, archivos txt, carpeta compartida con solo un rol de auditor) que permita la ejecución de los respectivos procedimientos. La documentación deberá ser ratificada posteriormente con la documentación original. Deberá tomar los recaudos correspondientes respecto a las afirmaciones de existencia y propiedad cuando se hayan definido la utilización de herramientas como videollamadas para realizar la inspección visual, resguardando, adecuadamente, dicha información. El contador incluirá, cuando corresponda, párrafos específicos en la carta de representaciones de la gerencia (FACPCE, 2020).

\section{d. Inspecciones oculares}

El auditor se puede encontrar con la imposibilidad de llevar a cabo inspecciones oculares de bienes de cambio o bienes de uso, procedimiento destinado a comprobar sus condiciones de venta o de uso y validar la razonabilidad de su valuación al cierre considerando su estado. Ante esta situación, el audi- 
tor podrá: utilizar el trabajo de expertos/especialistas no comprendidos en el Aislamiento Social Preventivo Obligatorio (ASPO) si la entidad continúa funcionando; solicitar al cliente material fotográfico del bien en cuestión, filmaciones, imágenes, manuales, folletería, etc. El auditor deberá: documentar adecuadamente el procedimiento seguido, y guardar los archivos que contengan dicho material, ratificar posteriormente, en su caso, con la inspección ocular del bien de cambio o de uso, según corresponda, e incluir - si lo considera pertinente - párrafos específicos en la carta de representaciones de la gerencia (FACPCE, 2020).

\section{e. Obtención de confirmaciones directas de terceros}

Continuando con el análisis de la "Guía sobre la aplicación de Normas de Contabilidad y Auditoría en el contexto COVID-19" (FACPCE, 2020) se prevé que el auditor podría encontrarse con restricciones en la recepción de respuestas del proceso de confirmación de saldos (circularización) de bancos, abogados, clientes y proveedores, así como otras confirmaciones de terceros. Ante esta situación, el auditor podrá: utilizar su e-mail para incrementar envíos de confirmaciones de saldos y recepción de respuestas con la correspondiente ratificación de la legitimidad de la dirección del e-mail de origen; documentar adecuadamente que la respuesta proviene del remitente objeto de la circularización y que quien suscribió la respuesta es el responsable de la temática consultada. Cuando el auditor lo considere conveniente ratificará posteriormente, con respuesta en copia física. Otra opción es observar cada una de las cuentas bancarias, préstamos, inversiones, etc., mediante el ingreso de un responsable de la entidad a la banca empresa y posterior envío de la documentación; cambiar excepcionalmente partidas de la muestra debido a la impracticabilidad de obtención de respuestas, considerando en futuras auditorías la reincorporación de esas partidas.

Otra situación que podría darse es la imposibilidad del tercero de responder solicitudes de confirmación de saldos por no tener acceso a la documentación fuente para poder informar los saldos requeridos. Ante esta situación, el auditor podrá indagar con la entidad acerca de la posibilidad de que los terceros (clientes, proveedores, otros) con quienes se vincula puedan acceder a sus sistemas mediante conexiones remotas o cuenten con sus sistemas almacenados dentro de una nube de datos de modo que puedan contar con la información necesaria para dar respuesta a los requerimientos. Otro procedimiento es soli- 
citar al cliente documentación por vía digital para llevar a cabo procedimientos alternativos tales como revisión de cobros/pagos posteriores o revisión de remitos conformados/avisos de recepción. Toda la información deberá ser adecuadamente documentada y en su caso ratificada posteriormente, con la documentación física que corresponda.

\section{f. Comprobaciones globales de razonabilidad y revisiones conceptuales}

$\mathrm{Al}$ ejecutar estos procedimientos, el auditor debe tener permanentemente presente los efectos que la pandemia y las medidas tomadas deberían haber tenido sobre las cuentas objeto de su revisión. El auditor deberá aumentar su escepticismo profesional al evaluar las explicaciones que reciba de la dirección y de personal de la entidad de las variaciones de las principales cifras de los estados contables. Puede ser de mucha utilidad comparar la información de la entidad con la de otras entidades de la industria para analizar patrones de comportamiento (benchmarking), dado que es esperable que la pandemia haya afectado de un modo similar a empresas de un mismo sector (FACPCE, 2020).

\section{g. Comprobación de la información relacionada}

En un contexto tan cambiante, es imperioso que la revisión de hechos posteriores al cierre del período y hasta la fecha del informe sea minuciosa, incrementando el escepticismo, poniendo especial énfasis en las nuevas medidas gubernamentales y las reacciones de la entidad y de la industria en general. El contador deberá evaluar que los hechos subsecuentes significativos tengan el adecuado tratamiento tanto en las mediciones de activos y pasivos como en las revelaciones en notas a los estados financieros anuales/intermedios objeto de auditoría/revisión. El auditor podría encontrarse con restricciones en la disponibilidad de información para el análisis de eventos posteriores. Ante esta situación, el auditor podrá solicitar al cliente documentación por vía digital que permita la ejecución de los respectivos procedimientos; ratificar posteriormente, en su caso, con la documentación original pertinente e incluir según corresponda, párrafos específicos en carta de representación (FACPCE,2020). 
Auditoría de estados financieros en contexto de pandemia por COVID-19: ...

\section{h. Examen de documentos importantes}

A través de la lectura de documentos tales como las actas de los órganos de dirección y administración, el contador identificará decisiones que puedan haberse tomado para paliar los efectos negativos de la pandemia. Deberá prestar atención a decisiones que se hayan adoptado con respecto a la discontinuación de segmentos de operación, reducción de jornadas laborales, potencial despido de personal, dificultades para obtener fuentes de financiamiento adicional, y otras decisiones de similar tenor que puedan tener impacto en los estados financieros y en la evaluación de la capacidad de la entidad para continuar como una empresa en marcha. En especial, deberá constatar que se hayan cumplido los requisitos legales dictados por los reguladores para las reuniones no presenciales, como consecuencia del ASPO. Verificará, asimismo, que se encuentren transcriptas en los registros, o resguardadas en otras clases de archivos, las actas de todas las reuniones realizadas. Ante esta situación, el auditor podría encontrarse con problemas de legalidad de decisiones tomadas en reuniones de directorio y asambleas celebradas a distancia, sin estar previsto en los estatutos sociales.

En consecuencia, según FACPCE (2020), el auditor podrá recomendar filmar, grabar o respaldar en algún medio magnético la reunión de Directorio en la que tuvo lugar la aprobación de los estados financieros objeto del encargo, conservar el video o audio como medio de prueba y ratificar posteriormente, mediante reunión presencial, las decisiones tomadas en reuniones de directorio y asambleas celebradas a distancia. Asimismo, podrá solicitar al síndico societario, en su caso, confirmación de la legalidad de la reunión de directorio o asamblea en la que estuvo presente. Cuando corresponda, podrá incluir párrafos específicos en la carta de representaciones de la gerencia.

\section{i. Obtención de una confirmación escrita de la dirección del ente de las expli- caciones e informaciones suministradas (manifestaciones de la dirección)}

Será importante para el auditor incluir en este documento manifestaciones sobre los efectos de la pandemia y las medidas tomadas, la evaluación de la dirección sobre la capacidad de la entidad para continuar como una empresa en marcha y sus planes para seguir operando, el adecuado cumplimiento de leyes y regulaciones, las condiciones de recupero de sus activos más significativos, y otras afirmaciones que sea conveniente que la dirección manifieste al 
auditor por escrito. El auditor podría encontrarse con dificultades para obtener una carta de representaciones de la gerencia debidamente firmada, o bien con dudas sobre la validez de la firma digital o electrónica. Ante esta situación, el auditor podrá: solicitar al cliente que el responsable firmante de la misma anticipe dicha carta directamente al auditor desde su mail institucional personal, con el compromiso de una ratificación posterior con firma ológrafa. Es altamente recomendable que el texto de la carta esté replicado en el cuerpo del e-mail, no solo en el archivo adjunto y recomendar el uso de firma digital en lugar de la ológrafa (FACPCE, 2020).

\subsection{Efectos en el informe del auditor}

En función de lo descripto anteriormente, pueden presentarse distintas situaciones derivadas de la pandemia que el auditor deberá evaluar al momento de emitir su opinión en el informe de auditoría. Las mismas pueden agruparse en incertidumbres significativas con respecto a la capacidad de la entidad para continuar como empresa en marcha y limitaciones en el alcance de la tarea del auditor (FACPCE, 2020).

La existencia de incertidumbres significativas de empresa en marcha es una de las principales cuestiones que deberá analizar el auditor y que tendrá impacto en la opinión. A tal efecto, deberá considerar: si existe incertidumbre significativa sobre empresa en marcha y fue revelada o no en nota a los estados contables; si la situación de pandemia impactó o impactará significativamente en el negocio del cliente y si la situación fue revelada o no en notas a los estados contables; si no hay elementos de juicio válidos y suficientes que indiquen que la entidad podrá continuar como empresa en marcha dentro de un futuro próximo en cuyo caso el auditor deberá revisar si la empresa utilizó, o no, criterios correspondientes a una empresa en funcionamiento para elaborar los estados contables.

Tanto la RT n. 37 (FACPCE, 2013) como la ISA 706 (IAASB, 2019) prevén la inclusión de un párrafo de énfasis en el informe del auditor referido a una materia revelada o presentada de manera apropiada en los estados financieros, que sea de importancia tal que resulte fundamental para el entendimiento que los usuarios tengan de la información. Entre las cuestiones que la normativa considera que deben incluirse en un párrafo de énfasis se encuentran las situaciones que afecten el principio de empresa en marcha (ISA 570 [IAASB, 2015]), situaciones cuya resolución dependa de sucesos futuros 
que no pueden ser previstos de manera razonable, descripción en nota de hechos posteriores al cierre de ejercicio que siendo significativos no requieren ajuste de las cifras de los estados financieros (ISA 560 [IAASB, 2010]), y la aplicación de una nueva norma contable que tiene efectos generalizados en los estados financieros (Lattuca, 2014). Si el auditor obtuvo elementos de juicio válidos y suficientes sobre la idoneidad de la utilización por parte de la dirección del principio de empresa en marcha y la situación se encuentra adecuadamente revelada en nota a los estados contables, expondrá la situación en el párrafo de énfasis sin modificar su opinión. Cuando la hipótesis de empresa en marcha no se encuentre revelada de manera adecuada se emitirá una opinión modificada por falta de exposición.

La otra cuestión que puede presentarse al auditor es que hubiese tenido limitaciones en el alcance de su trabajo. La RT n. 37 (FACPCE, 2013) establece que cuando el contador no hubiese podido obtener elementos de juicio válidos y suficientes que respalden su opinión pero concluya que los posibles efectos sobre los estados financieros de las incorrecciones no detectadas, si las hubiere, podrían ser significativos pero no generalizados, emitirá una opinión modificada. Si las limitaciones al alcance de la tarea de auditoría imposibilitaran la emisión de una opinión el auditor se abstendrá expresamente de opinar. En ambos casos, dichas limitaciones deben ser claramente expuestas en el apartado "Fundamentos de la opinión con salvedades", señalando claramente los procedimientos no aplicados y si la restricción ha sido impuesta por el contratante del encargo de auditoría, o es consecuencia de otras circunstancias.

Por último, cabe mencionar que el auditor podrá, si correspondiere, mencionar en un apartado del Informe sobre requerimientos legales y reglamentarios que la entidad no pudo actualizar sus libros contables indicando el período correspondiente. En la tabla 1 se exponen de manera sintética las diferentes situaciones derivadas de la pandemia que generan incertidumbre respecto a la continuidad de la empresa y su efecto en la opinión del auditor. 
Tabla 1. Incertidumbre sobre empresa en funcionamiento

\begin{tabular}{|c|c|c|c|}
\hline Problema & $\begin{array}{l}\text { Revelación } \\
\text { en notas a } \\
\text { los estados } \\
\text { financieros }\end{array}$ & $\begin{array}{l}\text { Inclusión } \\
\text { de un } \\
\text { párrafo de } \\
\text { énfasis }\end{array}$ & $\begin{array}{l}\text { Opinión } \\
\text { del auditor }\end{array}$ \\
\hline $\begin{array}{l}\text { Incertidumbre significativa sobre } \\
\text { empresa en marcha }\end{array}$ & Sí & Sí & Opinión sin modificar \\
\hline $\begin{array}{l}\text { Incertidumbre significativa sobre } \\
\text { empresa en marcha }\end{array}$ & No & No & $\begin{array}{l}\text { Opinión con salvedad por } \\
\text { falta de exposición }\end{array}$ \\
\hline $\begin{array}{l}\text { La pandemia impactó o impactará } \\
\text { significativamente en el negocio }\end{array}$ & Sí & Sí & Opinión sin modificar \\
\hline $\begin{array}{l}\text { La pandemia impactó o impactará } \\
\text { significativamente en el negocio }\end{array}$ & No & No & $\begin{array}{l}\text { Opinión con salvedad por } \\
\text { falta de exposición }\end{array}$ \\
\hline $\begin{array}{l}\text { No existen evidencias respecto } \\
\text { a la continuidad del ente como } \\
\text { empresa en marcha. EECC no pre- } \\
\text { parados con criterios de empresa } \\
\text { en marcha }\end{array}$ & Sí & Sí & Opinión sin modificar \\
\hline $\begin{array}{l}\text { No existen evidencias respecto } \\
\text { a la continuidad del ente como } \\
\text { empresa en marcha. EECC prepa- } \\
\text { rados con criterios de empresa en } \\
\text { marcha }\end{array}$ & No & No & Opinión adversa \\
\hline $\begin{array}{l}\text { El auditor tuvo limitaciones en el } \\
\text { alcance de su trabajo para obtener } \\
\text { evidencias sobre el principio de } \\
\text { empresa en marcha. Efecto signi- } \\
\text { ficativo y no generalizado }\end{array}$ & $\mathrm{N} / \mathrm{A}$ & No & $\begin{array}{l}\text { Opinión con salvedades } \\
\text { por los posibles efectos } \\
\text { sobre los EECC de las } \\
\text { incorrecciones no detec- } \\
\text { tadas }\end{array}$ \\
\hline $\begin{array}{l}\text { El auditor tuvo limitaciones en el } \\
\text { alcance de su trabajo para obtener } \\
\text { evidencias sobre el principio de } \\
\text { empresa en marcha. Efecto signifi- } \\
\text { cativo y generalizado }\end{array}$ & $\mathrm{N} / \mathrm{A}$ & No & Abstención de opinión \\
\hline
\end{tabular}

Fuente: elaboración propia.

\section{Consideraciones finales}

Sin lugar a dudas, la declaración del brote de COVID 19 como pandemia por la OMS impactó de manera muy significativa en la economía de las organizaciones presentando nuevos riesgos de negocios que deberán ser considerados por los auditores, especialmente en cuanto a la posibilidad de continuar como 
empresa en marcha y las posibles limitaciones para obtener elementos de juicio válidos y suficientes durante el desarrollo de su trabajo.

Desde un enfoque de auditoría basada en riesgos, el presente trabajo pretende identificar los eventos vinculados al negocio del cliente y al proceso de auditoría - incluyendo el riesgo de fraude - que deberán considerar los auditores de estados financieros durante la planificación y desarrollo de un encargo de auditoría y revisión, y el efecto de dichos eventos sobre el informe. Para ello se tuvo en cuenta la "Guía sobre la aplicación de Normas de Contabilidad y Auditoría en el contexto COVID-19" (FACPCE, 2020) en la cual se brindan pautas para el tratamiento de aspectos contables y de auditoría afectados por el contexto, como también las normas de contabilidad y auditoría vigentes en la República Argentina ya que la mencionada guía no conforma nueva normativa sino una orientación para la aplicación de la existente. Además de ello se consideraron las disposiciones de los ISA emitidos por el IAASB.

A pesar de la difícil situación que atraviesan las organizaciones es relevante sostener la calidad de la información contable generada por las organizaciones al igual que el trabajo del auditor quien deberá mantener al escepticismo profesional durante el desarrollo de los encargos y al momento de emitir su opinión. Al respecto, el impacto en el informe del auditor del incremental de riesgos asociados al contexto, desarrollados en los apartados anteriores, puede agruparse en incertidumbres significativas con respecto a la capacidad de la entidad para continuar como empresa en marcha y limitaciones en el alcance de la tarea del profesional.

En estudios posteriores se pretende obtener información empírica sobre los riesgos que debieron afrontar los auditores que se desempeñan en estudios de la Argentina, en el desarrollo de los encargos de auditoría de estados financieros cerrados a partir de la declaración de la pandemia.

\section{Referencias}

Albanese, D., Bonifazi, M. (2019). Revelación del Principio de Empresa en marcha Tratamiento en el informe del Auditor. Revista Enfoques, 11, 99-111.

Albanese D., López M. A. (2017). El entendimiento del negocio: pilar fundamental para una auditoría basada en riesgos. Caso de aplicación. DEG Profesional \& Empresaria, 215, 783-792.

Arens A., Elder, R. \& Beasley M. (2007). Auditoria. Un enfoque integral. México: Pearson, Prentice Hall. 
Bell, T., Marrs, F. O., Solomon, I., \& Thomas, H. (1997). Auditando organizaciones mediante una perspectiva estratégica de sistemas. En T. Bell, M. E. Peecher, I. Solomon, F. O. Marrs, H. Thomas. Auditoría basada en riesgos. Perspectiva estratégica de sistemas. Mantilla Blanco, S. A. (Trad.). Bogotá: Ecoe Ediciones.

Casal, A. M. (2013). La auditoría basada en riesgos y las nuevas normas de la Resolución Técnica (FACPCE) 37. Revista Desarrollo y Gestión, XIV(168), 955-977.

Casal, A. M. (2010). Gobierno Corporativo: dirección, administración y control de organizaciones de forma ética y responsables. Buenos Aires: Errepar.

International Accounting Standards Board (2008). Norma Internacional de Contabilidad (NIC) 1: Presentación de Estados Financieros. Recuperado de http:/ / www.facpce.org.ar:8080/miniportal/archivos/nic/NIC01.pdf

Federación Argentina de Consejos Profesionales de Ciencias Económicas (2000). Resolución Técnica N ${ }^{o}$ 17: Normas contables profesionales: Desarrollo de cuestiones de aplicación general y sus modificatorias. https:/ / www.facpce. org.ar/NORMASWEB/index_argentina.php?c $=1 \& s c=1 \& p=3$

Federación Argentina de Consejos Profesionales de Ciencias Económicas (2013). Resolución Técnica No 37. Normas de Auditoría, Revisión, Otros Encargos de Aseguramiento, Certificación y Servicios Relacionados. Disponible en http://www.facpce.org.ar:8080/NORMASWEB/index_argentina.php?$\mathrm{c}=1 \& \mathrm{sc}=1$

Federación Argentina de Consejos Profesionales de Ciencias Económicas (2015). Resolución Técnica No 41: Normas contables profesionales. Desarrollo de cuestiones de aplicación general: aspectos de reconocimiento y medición para entes pequeños. Recuperado de https:/ / archivo.consejo.org. ar/elconsejo/documentos/RT_FACPCE_N41.pdf

Federación Argentina de Consejos Profesionales de Ciencias Económicas (2020) Guía sobre la aplicación de las normas de contabilidad y auditoría frente a los efectos del Covid-19. Recuperado de https:/ / www.facpce.org.ar/wp-content/ uploads/2020/05/GuiaCovid-19-2.pdf

Hernández Melendrez, E. \& Sánchez Gómez, A. (2016). La supervisión. Auditoría y Control Interno, disponible en http:/ / alec.com.mx/uploads/links/94/ U3.3._174_La_supervision_-_GestioPolis_(2012-04-07_23-38-09).htm

International Auditing and Assurance Standards Board (2010). ISA 560 (revisada) Hechos posteriores al cierre. Recuperado de https://www.iaasb.org/ publications/basis-conclusions-isa-560-redrafted-subsequent-events

International Auditing and Assurance Standards Board (2013). ISA 240. Responsabilidades del auditor en relación con el fraude en una auditoria de estados financieros. Recuperado de https:/ / www.iaasb.org/publications/basis-con- 
Auditoría de estados financieros en contexto de pandemia por COVID-19: ...

clusions-isa-240-auditors-responsibilities-relating-fraud-audit-financial-statements

International Auditing and Assurance Standards Board (2015). ISA 570. Empresa en funcionamiento. Recuperado de https://www.iaasb.org/publications/international-standard-auditing-isa-570-revised-going-concern-3

International Auditing and Assurance Standards Board (2017). ISA N 540 (revisada). Auditoria de estimaciones contables y de la información relacionada a revelar. Recuperado de https://www.iaasb.org/publications/nia-540-revisada-auditor-de-estimaciones-contables-y-de-la-informaci-n-relacionada-revelar-0

International Auditing and Assurance Standards Board (2019). ISA 315 (revisada). Identificación y evaluación de los riesgos de incorrección material. Recuperado de https:/ / www.ifac.org/system/files/publications/files/ISA-315Full-Standard-and-Conforming-Amendments-2019-.pdf

International Auditing and Assurance Standards Board (2019). Norma internacional de Auditoría N 706 (revisada). Párrafos de énfasis y párrafos sobre otras cuestiones en el informe emitido por un Auditor independiente. Recuperado de https:/ / www.iaasb.org/publications/international-standard-auditing-isa-706-revised-emphasis-matter-paragraphs-and-other-matter-4

International Organization for Standardization (2009). ISO 31000. Risk Management - Principles and Guidelines. Suiza: ISO.

Latucca, A. J. (2014) Auditoría. Conceptos y Métodos. Rosario: Editorial Foja Cero. Mantilla Blanco, S.A. \& Casal, A. M. (2012 noviembre). Estándares internacionales de Auditoría. Cambios sustanciales y desarrollos recientes. Revista Desarrollo y Gestión, XIII(158), 1159-1182.

Muñoz Giró, J. (2011). El riesgo: su naturaleza y su gestión dentro de la organización. XVI Congreso Nacional de Auditores Internos del Sector Público. Costa Rica. Disponible en http://jaguar.cgr.go.cr/content/dav/jaguar/Documentos/cenrel/XVI_Congreso_AI/Doc_JMunozG.htm

Schultz, J. J. Jr., Bierstaker, J. L., \& O’Donell, E. (2010). Integrating business risk into auditor judgement about the risk of material misstatement. The influence of a strategic-systems-audit approach. Accounting, Organization and Society, 35, 238-251.

(C) 2021 por los autores; licencia otorgada a la revista Escritos Contables y de Administración. Este artículo es de acceso abierto y distribuido bajo los términos y condiciones de una licencia Atribución-No Comercial 4.0 Internacional (CC BY-NC 4.0) de Creative Commons. Para ver una copia de esta licencia, visite https://creativecommons.org/licenses/by-nc/4.0/ 